\title{
Engineered three-electrode cells for improving solid state batteries
}

\author{
Romain Dugas $^{1,2}$, Yves Dupraz ${ }^{3}$, Elisa Quemin ${ }^{1,2}$, Tuncay Koç ${ }^{1,2}$ and Jean-Marie \\ $\operatorname{Tarascon}^{1,2 *}$ \\ 1: Chimie du Solide et Energie-UMR CNRS 8260, Collège de France, Paris, France. \\ 2: Réseau sur le Stockage Electrochimique de l'Energie, FR CNRS 3459, Amiens, \\ France. \\ 3: Center for Interdisciplinary Research in Biology (CIRB), College de France, CNRS, \\ INSERM, Université PSL, Paris, France. \\ * jean-marie.tarascon@college-de-france.fr
}

\section{Abstract}

A three electrode cell for use with solid electrolytes is presented. The cell provides a stable reference voltage and leads artefact-free impedance spectra while being easily assembled and air-tight. To prove its versatility several combinations of electrodes and electrolytes were employed, enabling to rapidly assess the main differences between argyrodite vs. lithium phosphorous sulphide electrolytes, oxide vs. sulphide active materials or Li-In alloy based anode vs. Li foil anode. This new cell design offers a simple and efficient way of identifying the proper electrode-electrolyte components for enhancing solid state battery performances.

\section{Introduction}

All solid-state devices have the potential to bring the energy and power densities beyond the physical limits of the present Li-ion technology relying on liquid electrolytes ${ }^{1,2}$. Therefore, a massive effort is currently devoted to the processing of such batteries $^{3}$. Understanding and solving some of their specific issues, dealing with the use of solid state electrolytes such as cracks propagation, lithium dendrites formation or the reactivity and formation of space charge layers at the interface between electrolyte and 
active material, when an oxide is used in combination with a sulphide, are becoming crucial.

To date most electrochemical testing of all solid state batteries is performed in two electrodes configuration where the same electrode, namely the anode made of either pure $\mathrm{Li}$ or a biphasic Li-alloy $\left(\mathrm{Li}_{\mathrm{x}} \mathrm{M}_{\mathrm{y}}\right)$, serves both purposes of passing the current and providing a voltage reference. Such a setup limits the possibility to study precisely the phenomena occurring at a given electrode, since the voltage measured in a two electrode cell encompasses a contribution from both the anode and the cathode. To discriminate anodic processes from cathodic processes, a third reference is required as voltage reference through which no current is flowing upon cell operation.

In a liquid electrolyte cell, the third electrode is typically a thin wire located within the wetted separator placed between the anode and the cathode. Solid state batteries have a different configuration. They can be viewed as a single cylinder, resulting from the pressure stacking of electrodes and electrolytes pellets, placed between two pistons sliding inside a cylinder for testing. Introducing a third electrode in such a battery is complicated by the pressure required to assemble the cell, which will easily break a thin wire. It also requires to extract the wire from the cell to take an electrical contact, either through a tiny hole in the edge of the cylinder or between the cylinder and one of the pistons. Furthermore, in solid-state batteries a crucial role is played by interfaces, for the analysis of which impedance spectroscopy is a technique of choice. The use of such technique brings supplementary constraints, especially regarding the symmetry of the cell, to avoid the appearance of artefacts on the impedance spectra ${ }^{4-6}$.

A few examples of all-solid-state three electrode cells can be found in the literature. On one hand, some of them consist in firstly assembling a two electrode cell, then adding a $\mathrm{Li}$ or $\mathrm{Li}-\mathrm{In}$ electrode to provide a stable reference potential ${ }^{7,8}$. No EIS in 3 
electrodes configuration was reported with such cells and it is probable that the asymmetric cell geometry or the position of the reference might bring artefact loops in the impedance spectra. On the other hand, EIS could be performed in 3 electrodes configuration using gold plated tungsten wire located between anode and cathode as reference ${ }^{9}$, which may not provide a stable voltage upon cycling ${ }^{10}$. More recently, a reference electrode made of a Ni mesh coated with lithium titanate allowed to follow the voltage of both electrodes, and also properly record their impedances ${ }^{11}$.

In this work we present an air-tight three-electrodes cell for electrochemical characterisation of battery materials with solid state electrolytes. The cell takes advantage of the sequential cold-pressing assembly procedure of the solid-state battery to easily build a reference element directly in the cell stack, without using a fragile wire or mesh. It results in an ideally axisymmetrical geometry suitable for electrochemical impedance spectroscopy. Various electrodes-electrolytes pairing were studied for proof of concept with the results enabling to rapidly identify the proper electrode-electrolyte pairing. Moreover, we show that this new engineered three-electrode cell helps in better understanding Li deposit and stripping phenomena.

\section{Experimental}

Cells were assembled with either $\mathrm{LiNi}_{0.6} \mathrm{Mn}_{0.2} \mathrm{Co}_{0.2} \mathrm{O}_{2}$ (NMC) or $\mathrm{TiS}_{2}$ (Aldrich) or home-made $\mathrm{Li}_{1.13} \mathrm{Ti}_{0.57} \mathrm{Fe}_{0.3} \mathrm{~S}_{2}{ }^{12}$. The active material was mixed with solid electrolyte in a 70:30 weight ratio, either by hand with mortar and pestle or by ball milling for $20 \mathrm{~min}$ with a Spex 8000M and a ball to powder ratio of 20:1.

Either $\beta$-Li $\mathrm{PS}_{4}$ ( $\beta$-LPS) or $\mathrm{Li}_{6} \mathrm{PS}_{5} \mathrm{Cl}$ solid electrolyte was employed, which were prepared by solution mediated ${ }^{13}$ and solid state synthesis ${ }^{14}$, respectively. 
A mixture of Li-In alloy and solid electrolyte was used as counter electrode (CE). The alloy was prepared by weighting Li and In foils (Sigma) in a 33.2 ratio to target $\mathrm{Li}_{0.5}$ In composition. The use of the $\mathrm{Li}_{0.5}$ In composition ensures that the anode voltage directly corresponds to the In/LiIn couple as the cell is assembled. Moreover, it provides a $\mathrm{Li}$ reservoir to ensure that the discharge is limited by the cathode. The $\mathrm{Li}$ was enfolded inside the In foil, then the foil was laminated and folded several times between a glass tube and a polyethylene sheet until the alloy became matt and brittle. To increase the electroactive surface area and prevent CE delithiation from limiting the discharge of the cells ${ }^{7}$, the alloy was mixed with electrolyte powder in a 60:40 weight ratio and mixed with mortar and pestle to obtain a dark grey powder. No attempt to optimize this mass ratio was made. A similar procedure was employed to prepare a $\mathrm{Li}_{0.9}$ In: $\beta$-LPS composite to be used as CE for cells assembled with $\mathrm{TiS}_{2}$.

The three electrodes cell was realised by modification of the two electrodes setup developed in our laboratory, and consists of a cylindrical polyetherimide (PEI) cell body and two stainless steel pistons of $8 \mathrm{~mm}$ diameter. The cell body was divided in two PEI parts and a stainless steel ring was included to take the contact of the reference electrode (RE). It is assembled by means of 6 screws and nuts; two o-rings are used to ensure air-tightness; and a piece of metal foil ( $15 \mu \mathrm{m} \mathrm{Al}$ in this work), in which an $8 \mathrm{~mm}$ hole is punched, is clamped in the middle to be used as RE current collector (see figure 1a). After assembly of the cell body an $8 \mathrm{~mm}$ PEI cylinder is obtained, with a ring shaped RE current collector which will be conveniently located at the level of the top of a piston in the blank cell.

The stainless steel pistons are part of two platens, which close the whole cell by means of a second set of 6 screws (figure 1b). Insulating inserts are placed under the screws' heads and standard Nylon ferrules provide air-tightness to the whole cell. The 
initial pressure is controlled by means of the gradually applied torque of the closing screws. The final value of $2.3 \mathrm{Nm}$ corresponds to a pressure of $1 \mathrm{t} \mathrm{cm}^{-2}$, as calibrated using a force sensor (Applied Measurements) in the blank cell.

The three electrodes cell is assembled as follows. As a preliminary step a solid electrolyte pellet is prepared in a die set by compressing $45 \mathrm{mg}$ of solid electrolyte at $4 \mathrm{t} \mathrm{cm}^{-2}$ for $3 \mathrm{~min}$. In a first step (figure $2 \mathrm{a}$ ) the RE composite material is added in the cell, then the solid electrolyte pellet and the second piston, and a pressure of 1 ton $\mathrm{cm}^{-2}$ is applied for a few seconds. After this step the RE layer is densified and the tension it applies on the wall of the cell maintains it at the level of its current collector. This procedure takes of advantage of the use of a solid electrolyte to avoid placing any wire or mesh inside the cell, which would be likely to be damaged by the assembling pressure. Instead, the contact between the RE and its current collector is taken at the periphery of the RE. In a second step (figure $2 \mathrm{~b}$ ) the cell is flipped and $40 \mathrm{mg}$ of electrolyte powder are added then pressed for a few seconds at $1 \mathrm{t} \mathrm{cm}^{-2}$ to create the second electrolyte layer. Finally, the working (WE) and counter electrode (CE) composite powders are added each at a side of the cell (figure 2c) and the whole assembly is pressed under $4 \mathrm{t} \mathrm{cm}^{-2}$ for $15 \mathrm{~min}$. The WE is placed in the side where the electrolyte layer is thinnest. After the 15 min compression, the cell is closed, taken out of the glove box and ready to cycle (figure $2 \mathrm{~d}$ ).

The composite RE material is a mixture of electroactive material versus which the potentials are recorded, and solid electrolyte. Since the RE layer is located in the middle of the cell, the addition of electrolyte in this layer is necessary to ensure sufficient $\mathrm{Li}^{+}$ conduction between working (WE) and counter (CE) electrodes. This composite RE takes the form of a layer located between WE and CE and covering the whole cell, leading to an ideal geometry for impedance spectroscopy similar to the one described 
in $^{15}$ for liquid electrolytes. In practice the same $\mathrm{Li}_{0.5} \mathrm{In}$ : solid electrolyte composite (60:40 w./w.) was used for both $\mathrm{RE}$ and $\mathrm{CE}$. Cells employing the $\mathrm{Li}_{0.9}$ In alloy composition as $\mathrm{CE}$ active material were also assembled with a $\mathrm{Li}_{0.5} \mathrm{In}$ : solid electrolyte RE layer.

To prepare the Li metal anode employed in a few cells, a piece of Li foil (Aldrich) was scratched and laminated with a glass tube to obtain a clean and flat surface from which an $8 \mathrm{~mm}$ diameter disc was punched out. Cells using this type of anode were placed in a specific frame equipped with a force sensor to apply a low pressure of $45 \mathrm{~kg} \mathrm{~cm}^{-2}$, and kept in the glove box for cycling.

Two electrode cells were assembled in similar cells except for the absence of a RE current collector. In this case the assembly is performed in two steps: 1) cold pressing of the electrolyte inside the cell; and 2) addition and cold pressing of the WE and CE.

Galvanostatic cycles were performed at room temperature at $\mathrm{C} / 25$ for sulphides and $\mathrm{C} / 20$ for NMCs, where $\mathrm{C}$ correspond to 1 mole of Li per mole of active material in $1 \mathrm{~h}$, using a VMP3 potentiostat/galvanostat (BioLogic) controlled with EC-Lab software. $\mathrm{Li}_{1.13} \mathrm{Ti}_{0.57} \mathrm{Fe}_{0.3} \mathrm{~S}_{2}$ was cycled between 1.2 and $2.4 \mathrm{~V}$ vs. LiIn/In, NMC between 2 and 3.6 V , and $\mathrm{TiS}_{2}$ between 0.8 and $2.4 \mathrm{~V}$. Impedance spectra were measured after $1 \mathrm{~h}$ rest using a $15 \mathrm{mV}$ sinusoidal perturbation. 10 points per decade were taken for frequencies $f$ of $200 \mathrm{kHz}$ to $10 \mathrm{mHz}$, plus 5 points between 10 and $1 \mathrm{mHz}$ so as to keep the recording of a spectrum below $75 \mathrm{~min}$. The spectra were fitted using the built-in module in ECLab.

The conductivity of solid electrolyte pellets was measured in two electrodes configuration in the $5 \mathrm{MHz}-1 \mathrm{~Hz}$ frequency range with MTZ impedance analyzer (BioLogic). 
All experiments were conducted at room temperature $\left(\sim 24{ }^{\circ} \mathrm{C}\right)$ and repeated at least twice.

\section{Results and discussion}

Three different solid electrolyte powders were employed in this work: two batches of $\beta$-LPS and one of argyrodite. Their complex resistivity is plotted in figure 3a) for the two former and in figure 3b) for the latter. It consists in a high frequency loop from which the resistivity of the pellet is deduced, followed by a quasi-vertical impedance increase corresponding to the geometrical capacitance of the cell. The $\beta$-LPS batches have comparable resistivity of 4,670 and $4,250 \Omega \mathrm{cm}$, corresponding to a conductivity in the order of $0.2 \mathrm{mS} \mathrm{cm}^{-1}$, in accordance with literature ${ }^{13}$. However, a significant difference is observed regarding the frequency at apex of the high frequency loop: $615 \mathrm{kHz}$ for batch one and $4.83 \mathrm{MHz}$ for batch two. Given the complex structure of the solid electrolyte prepared by solution-mediated route ${ }^{16}$, this is likely an indication that the composition in poorly conductive phases has low repeatability between the two batches and affects the permittivity of the material. This discrepancy will translate into different extents of the solid electrolyte loops in the impedance measurements, depending on the batch employed.

In figure $3 b$ ), argyrodite displays more than $15 \times$ lower resistivity than $\beta$-LPS. The corresponding conductivity of $3.7 \mathrm{mS} \mathrm{cm}^{-1}$ is in good agreement with previously reported values for this electrolyte ${ }^{14}$. With argyrodite the high frequency loop can barely be observed. From the few points measured at highest frequencies it can estimated that the apex is around $190 \mathrm{MHz}$, a frequency domain which cannot be investigated with our instruments. 
Preliminary to the measures in three electrode configuration, the procedure to prepare WE and CE was optimized in two electrodes configuration using $\mathrm{Li}_{1.13} \mathrm{Ti}_{0.57} \mathrm{Fe}_{0.33} \mathrm{~S}_{2}$ (LTFS) and Li-In alloy as benchmark materials. The performance of these materials in two electrodes configuration is reported in figure 4 for composite cathode materials prepared either by hand grinding or high energy ball milling. The alloy in the $\mathrm{CE}$ is $\mathrm{Li}_{0.5} \mathrm{In}$ as initial composition and is mixed with $\beta$-LPS solid electrolyte.

In the voltage profiles in figure 4a) and b) it can be observed that the first charge differs significantly from the subsequent ones which are $100 \mathrm{mV}$ lower and that the average discharge voltage slightly decays upon cycling (-30 mV in five cycles). Since the counter electrode is expected to remain at a constant voltage of $622 \mathrm{mV} v s . \mathrm{Li}^{+} / \mathrm{Li}^{17}$, these features can be attributed to the LTFS cathode material. Indeed, they are the typical response of materials based on anionic redox activity and the voltage profiles are in good accordance with the ones measured in liquid organic electrolytes ${ }^{12}$.

At first charge, $0.7 \mathrm{Li}$ are extracted per formula unit of LTFS in the hand ground composite (figure 4a)), leading to a capacity of $160 \mathrm{~mA} \mathrm{~h} \mathrm{~g}^{-1}$. The capacity slightly increases for subsequent cycles but remains below $180 \mathrm{~mA} \mathrm{~h} \mathrm{~g}^{-1}$. In contrast, figure $4 \mathrm{~b}$ ) shows that almost $95 \%$ of the Li can be extracted from the ball milled composite at first charge, without increase in capacity upon cycling. The reversible capacity is $243 \mathrm{~mA} \mathrm{~h} \mathrm{~g}^{-1}$ at first cycle, $35 \%$ higher than the maximum obtained with the hand ground composite.

As seen in figure $4 \mathrm{c}$ ), the reversible capacity of the hand ground composite is maximised at fourth cycle, and slightly decreases by $3.7 \%$ between cycles 4 and 10 . The ball milled composite, which has maximum capacity at $1^{\text {st }}$ cycle, displays a comparable capacity retention with $4.6 \%$ loss at $7^{\text {th }}$ cycle. The capacity increase of the 
hand ground electrode suggests that a higher amount of active material becomes accessible to both $\mathrm{e}^{-}$and $\mathrm{Li}^{+}$ions, since a hand ground composite displays full capacity at $1^{\text {st }}$ cycle with a liquid electrolyte ${ }^{12}$. The mechanism for such activation with a solid electrolyte is unclear; it possibly involves local deformation of the electrolyte in the cathode composite as a consequence of the pressure variation upon cycling, which could slightly improve the double percolation.

A very good coulombic efficiency is obtained for LTFS vs. $\mathrm{Li}_{0.5} \mathrm{In}$ : more than $97.5 \%$ since the first cycle, regardless of the method employed to prepare the composite cathode material. This contrasts with the efficiency lower than $70 \%$ obtained at first cycle with the same cathode material in combination to an anode consisting only of $\mathrm{Li}$ and $\mathrm{In}$ metals ${ }^{18}$. This comparison shows that a purely metallic anode limits the cell's discharge and emphasises the benefit of mixing the alloy with solid electrolyte powder, in good agreement with ref $^{7}$. Consequently the $\mathrm{Li}_{0.5} \mathrm{In}$ :electrolyte composite $\mathrm{CE}$ was employed in the following.

Several combinations of active materials and electrolytes were tested in three electrodes configuration with $\mathrm{Li}_{0.5} \mathrm{In}$ alloy as reference. Galvanostatic charges and discharges were stopped every two hours to record impedance spectra after a resting period. Figure 5 displays the first two cycles for both WE and CE recorded for various combinations of WE active material and solid electrolyte. In the case of ball-milled LTFS and $\beta$-LPS (figure 5a), the reversible capacity is $233 \mathrm{~mA} \mathrm{~h} \mathrm{~g}^{-1}$ and the voltage profile is comparable to that observed in two electrodes configuration, with exception of the vertical lines due to the addition of resting periods in the cycling protocol.

A simple transposition to NMC (figure 5b) leads to very poor performance: the reversible capacity is no more than $60 \mathrm{~mA} \mathrm{~h} \mathrm{~g}^{-1}$, with only $40 \%$ coulombic efficiency at first cycle. An important initial sloping region between 2.3 and $3.1 \mathrm{~V}$ is noticed at 
first charge. This slope was previously attributed to electrolyte decomposition, which was enhanced in the presence of carbon as conductive additive in the cathode composite $^{19}$. Since no carbon was included in the present case, it suggests that the reactivity between active material and electrolyte is increased by the high energy ball milling. Indeed, with a composite prepared by hand grinding (figure $5 \mathrm{c}$ ) the initial slope extends for a much shorter capacity (2.3 $\mathrm{mA} \mathrm{h} \mathrm{g}^{-1}$ instead of 27), leading to a significantly higher efficiency $(64 \%)$ though the reversible capacity remains at the same low value of $60 \mathrm{~mA} \mathrm{~h} \mathrm{~g}^{-1}$. The polarization is also lowered, especially upon reduction. Nevertheless, this polarization remains in the order of $\pm 150 \mathrm{mV}$ at $\mathrm{C} / 20$, which appears detrimental for practical application.

Turning to the $\mathrm{Li}_{6} \mathrm{PS}_{5} \mathrm{Cl}$ argyrodite, the polarization is lowered by a factor 3, as expected given its more than 10 times higher conductivity and the first charge capacity is drastically improved reaching $140 \mathrm{~mA} \mathrm{~h} \mathrm{~g}^{-1}$ (figure $5 \mathrm{~d}$ ). Only $108 \mathrm{~mA} \mathrm{~h} \mathrm{~g}^{-1}$ can be recovered on the following discharge leading to a $77 \%$ efficiency. The missing $33 \%$ irreversible capacity ( $\left.31 \mathrm{~mA} \mathrm{~h} \mathrm{~g}^{-1}\right)$ is similar to the one measured for the hand ground NMC: $\beta$-LPS composite, suggesting that this loss is intrinsic to the active material itself, rather than coming from irreversible side reactions. However, in contrast to the NMC: $\beta$-LPS cells, the second cycle superimposes perfectly with the first one, without any measurable slippage at end of charge and end of discharge, hence indicating a nice reversibility of the system.

Regarding the $\mathrm{Li}_{0.5}$ In counter electrode, figure 5 shows that its redox potential remains in a potential window of $\pm 160 \mathrm{mV}$ around the $\mathrm{RE}$ with a polarization that slightly changes owing to some inhomogeneity of composite CE. However, at this stage all attempts to better homogenize the composite by ball milling or Vortex mixing, led to a poor CE performance.-The situation drastically changes when argyrodite is used as 
counter electrode since the voltage window narrows down from $\pm 160 \mathrm{mV}$ to $\pm 20 \mathrm{mV}$ so that a 2 electrodes configuration can safely be employed with this electrolyte $(20 \mathrm{mV}$ correspond to $\leq 2 \mathrm{~mA} \mathrm{~h} \mathrm{~g}^{-1}$ at end of charge for NMC).

The impedance spectra recorded for the various working electrodes in the middle of second discharge (symbols in figure 5) are reported in figure 6 for the different systems of interest. They appear free of artefact loops which might extend until positive values of $\operatorname{Im}(Z)^{15}$, as expected based on the axial symmetry of the cell. All impedance spectra were fitted with the response of an equivalent circuit made of resistors (R) and constant phase elements (CPE). Various parallel R-CPE elements were assembled in series with a last CPE (reflective boundary conditions). The impedance of a CPE was taken as $\mathrm{Z}_{\mathrm{CPE}}=1 /\left[Q(j 2 \pi f)^{\alpha}\right]^{20}$, where $j=\sqrt{ }(-1)$, and $\alpha$ characterises either the slope in a Nyquist plot of the linear response of a single CPE, or the depression of the semicircular response in case of an R-CPE element. The CPE factor $Q$ determines the position in the frequency domain; in case of a parallel R-CPE circuit, the apex of the circular arc is at $f_{0}=1 /\left[2 \pi(\mathrm{R} Q)^{1 / \alpha}\right]^{21}$.

In the impedance spectrum of the LTFS: $\beta$ LPS composite is shown in figure 6 a with the solid electrolyte signature being clearly identified as an almost perfectly semicircular loop $(\alpha=0.98)$. The $283 \Omega \mathrm{cm}^{2}$ resistance of this loop corresponds to a conductivity of $1.9 \mathrm{mS} \mathrm{cm}{ }^{-1}$ and the frequency at apex is $f_{0}=340 \mathrm{kHz}$. These values are in good accordance with those of a conductivity measurement of the same electrolyte in 2 electrodes configuration that shows both a $14 \%$ lower conductivity and a $10 \%$ higher value of $\log \left(f_{0}\right)$. The rest of the impedance spectrum can be well described by a second R-CPE loop and CPE in series. The latter corresponds to a $\sim 45^{\circ}$ slope in the Nyquist plot $(\alpha=0.53)$, which points at diffusion in the active material particles. The second R-CPE loop can therefore be attributed to charge transfer at the 
interface between solid electrolyte and active material in the WE composite, with possibly some contribution from $\mathrm{Li}^{+}$conduction in the cathode composite.

The impedance of the ball-milled NMC: $\beta$-LPS cathode composite (figure $6 \mathrm{~b}$ ) appears significantly higher, since its real part is more than $2.2 \mathrm{k} \Omega \mathrm{cm}^{2}$ at $f=1 \mathrm{mHz}$, a value four times higher than for LTFS. At highest frequencies, the response of the solid electrolyte is not as precisely defined as with LTFS. Therefore, the impedance of a pellet (figure $3 a$ ) of the corresponding mass was employed in the equivalent circuit, together with the addition of a second loop at $f_{0}=32 \mathrm{kHz}$ required to match the experimental data. Note that modifying the parameters of a single R-CPE loop cannot be satisfactory since such a loop would extend until negative values of $\operatorname{Re}(Z)$ for $f \rightarrow \infty$, and this lacks physical sense. Since the additional loop displays a relatively high $\alpha$ value $(0.85)$ in comparison to the ones observed at lowest frequencies and $\alpha=1$ for an ideal planar capacitor, we speculate that it might correspond to some process occurring at the planar interface between electrolyte layer and cathode composite. For decreased frequencies the impedance can be described with a R-CPE loop followed by a $45^{\circ}$ slope, similarly to the case of LTFS. But in contrast with LTFS, the R-CPE loop (apex at $f_{0}=12 \mathrm{~Hz}$ ) appears very broad, $12.5 \times$ more resistive than the one attributed to charge transfer of LTFS $\left(1750 \Omega \mathrm{cm}^{2}\right)$, and consequently the diffusion is barely observed at lowest frequencies.

In comparison to the ball-milled composite, the hand ground NMC: $\beta$-LPS composite has a lower impedance, the real part of which remains below $900 \Omega \mathrm{cm}^{2}$ for the investigated frequency range (figure $6 c$ ). This is in line with the lower polarization observed in figure 5. Alike the ball-milled composite, the impedance measured at highest frequencies is significantly higher $(\sim 50 \%)$ than the resistance due to the amount of solid electrolyte inserted between WE and RE in the cell. The solid 
electrolyte loop itself is barely visible due to the use of the second batch of $\beta$-LPS. Therefore, in the equivalent circuit the solid electrolyte properties were taken from the conductivity measurements in figure $3 a$ ), and a second loop attributed to the interface between electrolyte layer and cathode composite was also included in the equivalent circuit for consistency with both the solid electrolyte conductivity and the observation of this second loop with the ball-milled composite. At lowest frequencies, the $\sim 45^{\circ}$ slope cannot be observed, only an important loop is seen (apex at $f_{0} \simeq 30 \mu \mathrm{Hz}$, outside of the investigated range), suggesting very sluggish charge transfer at solid electrolyte/active material interface in the composite. In between, a complex response is observed for $15 \mathrm{kHz} \gtrsim f \gtrsim 20 \mathrm{~Hz}, \mathrm{Z}$ ?, consisting of a rather linear evolution of $\operatorname{Im}(\mathrm{Z}) v s \cdot \operatorname{Re}(\mathrm{Z})$ followed by a circular arc. Such response can be described either by a series of two R-CPE elements ${ }^{22}$ or an infinite transmission line which accounts for the distribution of active material along a resistive path ${ }^{23,24}$. At this stage, no experimental data allows to discriminate between the different options, and two simple R-CPE elements were employed only for the sake of simplicity.

Lastly, the use of argyrodite has a dramatic effect on the impedance (in figure 6d), which decreases by an order of magnitude as a direct consequence of the higher electrolyte conductivity. Since the solid electrolyte loop occurs at very high frequency ( $\sim 250 \mathrm{MHz}$ at apex), it is completely outside of the accessible frequency range of the potentiostat. However, in this case again the impedance measured at highest frequencies does not match the conductance of the electrolyte layer, $\left(15 \Omega \mathrm{cm}^{2}\right)$ indicating probable presence of a second high-frequency process. At high to intermediate frequencies $(150 \mathrm{kHz} \gtrsim f \gtrsim 35 \mathrm{~Hz})$, the same shape as with $\beta$-LPS is observed, which was again described by two R-CPE loops in the equivalent circuit. Finally, at lowest frequencies a last loop attributed to charge transfer is seen, followed 
by the typical $45^{\circ}$ slope due to $\mathrm{Li}^{+}$diffusion in the active material particles. In summary the response is qualitatively the same as with $\beta$-LPS (figure $6 c$ ), but the faster processes with argyrodite allow to observe the diffusion in NMC, which was not visible with $\beta$-LPS for the same frequency range.

Overall in figure 6 a gradual increase in complexity of the spectra is observed when moving from LTFS to NMC, and then from a ball-milled composite to a hand ground one. Obviously further investigation regarding NMC is needed and it ranges from testing more experimental conditions to a greater data analysis for unravelling the origin of the numerous features in the impedance spectra. Whatever, these complicated impedances highlight the problematic of mastering the interface between oxide type cathode materials and sulphide based solid electrolytes, and by the same token the need to develop strategies such as active material coatings to improve it ${ }^{25}$.

Figure 7 illustrates the benefit of the 3 electrodes configuration for the recording of impedance spectra. Figure 7a) displays the impedance of LTFS fitted with the same 3 elements circuit as previously. The impedance of the CE simultaneously recorded is reported in figure $7 b$ ). It shows a high frequency loop corresponding to $\mathrm{Li}^{+}$conduction in the solid electrolyte layer between RE and CE (dashed line), and two highly depressed loops at lower frequencies.

The impedance of a LTFS: $\mathrm{Li}_{0.5} \mathrm{In}$ cell in 2 electrodes configuration was reconstructed simply by summing the impedances of WE and CE. The contribution of the electrolyte between RE and CE (i.e., the dashed line in figure 7b)) was subtracted to simulate a cell with an electrolyte thickness equivalent to the one between WE and RE in the 3 electrodes cell. The resulting impedance spectrum is displayed in figure 7c) with open triangles. The equivalent circuit in figure 7a) was used to fit this spectrum as well, which lead a satisfactory result shown in figure 7c). This implies that the WE 
and CE impedances cannot be decorrelated in a 2 electrodes measurement. At lowest frequencies the WE contributes most to the 2 electrodes impedance, and therefore the Warburg parameter is in the same order as in 3 electrodes configuration $(20 \%$ discrepancy). However, the intermediate frequency loop is more significantly affected since its resistance is more than doubled ( $307 \Omega \mathrm{cm}^{2}$ instead of 139). Obviously the difference corresponds to the contribution of the $\mathrm{CE}$ and should not be attributed to the WE. Furthermore, if such a spectrum evolves during cycling there is no way to identify which electrode is at the origin of the variations.

Altogether, this survey indicates that the impedance of LTFS is probably the most simple as it can be fitted with the 3 elements equivalent circuit depicted in figure 6a). Figure 8a shows that this simple equivalent circuit is indeed adequate to fit the impedance spectra of an entire cycle despite significant changes in the spectra shown in insets. These changes are mostly attributable to the resistance of the loop appearing at intermediate frequencies, after the response of the solid electrolyte. Since the ion conductivity of $\beta$-LPS in the cathode composite is not expected to be dependent on the state of charge, variations in resistance of this loop can be attributed to the charge transfer to the active material, $\mathrm{R}_{\mathrm{CT}}$. This resistance varies by an order of magnitude over the course of the second cycle: during charge $R_{\mathrm{CT}}$ is in the range of 20 to $45 \Omega \mathrm{cm}^{2}$ and is minimized in the middle of charge, whereas during discharge a gradual increase is observed from 75 up to $230 \Omega \mathrm{cm}^{2}$.

In figure $8 b) R_{C T}$ is plotted as a function of charge and discharge capacity for the five first cycles, together with the potential of LTFS vs. the $\mathrm{Li}_{0.5} \mathrm{In} \mathrm{RE}$. A very good correlation is seen between $\mathrm{R}_{\mathrm{CT}}$ and the voltage. At first charge $\mathrm{R}_{\mathrm{CT}}$ remains at low values of $10-15 \Omega \mathrm{cm}^{2}$ while LTFS displays a single voltage plateau. For each discharge $\mathrm{R}_{\mathrm{CT}}$ gradually increases as seen in figure $8 \mathrm{~b}$ ). The maximum $\mathrm{R}_{\mathrm{CT}}$ value 
increases cycle by cycle, and reaches $1500 \Omega \mathrm{cm}^{2}$ at the end of 5 th cycle. After the $1^{\text {st }}$ cycle, the charge starts with a decrease in $\mathrm{R}_{\mathrm{CT}}$ which matches well the initial voltage slope between 1.5 and $1.9 \mathrm{~V} v$ s. LiIn:In, followed by a constant and lowest value of $\mathrm{R}_{\mathrm{CT}}$ corresponding to the plateau around $2 \mathrm{~V}$. Thus, $\mathrm{R}_{\mathrm{CT}}$ displays a strong asymmetry between charge and discharge, and its variations appear to match well the voltage variations of LTFS. The strong asymmetry suggests that it might be related with the differing reaction pathways between charge and discharge for processes owing to the anionic redox process pertaining to the Li-rich layered sulphide LTFS as well documented ${ }^{26,27}$.

To further evidence the origin of the variations of $\mathrm{R}_{\mathrm{CT}}$, we performed the same analysis with the classical sulphide-based electroactive material, $\mathrm{TiS}_{2}$, in which $\mathrm{Li}^{+}$ intercalation is a pure cationic process. The second cycle with this material and $\beta$-LPS as electrolyte is plotted in figure 6c). A gradual voltage decrease is observed upon $\mathrm{Li}$ intercalation in $\mathrm{TiS}_{2}$, as is well known in the literature ${ }^{28,29}$. The process has high reversibility above $99 \%$ (94\% at $1^{\text {st }}$ cycle). Several impedance spectra are shown in insets, which can also be well described with the same simple model accounting for electrolyte, charge transfer and diffusion. However, using $\mathrm{TiS}_{2} \mathrm{R}_{\mathrm{CT}}$ is significantly lower than with LTFS, and therefore a $60 \times 36 \Omega^{2} \mathrm{~cm}^{4}$ region around the end of the solid electrolyte loop is plotted, corresponding to frequencies above $\sim 30 \mathrm{mHz}$. The extent of that loop differs from figure 6a) because of the use of different $\beta$-LPS batches, as previously discussed. Unlike LTFS, $\mathrm{TiS}_{2}$ displays very similar impedance spectra between charge and discharge. Upon lithiation, $\mathrm{R}_{\mathrm{CT}}$ gradually decreases from $10-12 \Omega \mathrm{cm}^{2}$ down to less than 2.5 , and the effect is reversed upon delithiation. When comparing with $\mathrm{TiS}_{2}$, it can be noted (Fig. 8d) that the charge-discharge traces neatly superimposed during the first 5 cycles indicating an excellent reversibility (> $94 \%)$. 
These 5 cycles lasted more than $680 \mathrm{~h}$, which demonstrates a very good stability of the $\mathrm{Li}_{0.5}$ In: $\beta$-LPS RE. Unlike LTFS, the $\mathrm{R}_{\mathrm{ct}}$ for $\mathrm{TiS}_{2}$ remains below $20 \Omega \mathrm{cm}^{2}$ and it becomes lower upon cycling reaching values as low as $2.5 \Omega \mathrm{cm}^{2}$ (shaded area in figure 8d)) upon cycling. Values in this area are only indicative of a negligible $R_{c t}$ since the quality of the fit is not altered upon removal of the corresponding loop.

Finally, the influence of the anode was observed with LTFS cathode material and $\beta$-LPS electrolyte by moving from $\mathrm{Li}_{0.5} \mathrm{In}: \beta$-LPS (Figure 9) composite to a pure $\mathrm{Li}$ anode (Figure 10) while keeping a $\mathrm{Li}_{0.5} \mathrm{In}$ : $\beta$-LPS RE layer. Figures $9 \mathrm{~b}$ ) to e) report the impedance of the $\mathrm{Li}_{0.5} \mathrm{In}: \beta$-LPS anode during cycling (figure 9a). In each subfigure the impedance spectra are plotted with a regular time interval for a given sequence, lithiation or delithiation. The electrolyte resistance, $\mathrm{R}_{\mathrm{el}}$, is well identified in all spectra. Since it varies by $\pm 17 \%$ during the 1 st cycle and $\pm 3 \%$ during the 2 nd one, the spectra were centered on $R_{\mathrm{el}}$ to emphasize the variations at lower frequencies. During first lithiation (figure 9b), the impedance of the anode increases and the growth of a loop is observed at low frequencies (apex at $540 \mathrm{mHz}$ end of LTFS charge). This process keeps growing monotonously with time during the subsequent delithiation and lithiation as indicated by the arrow marks in figure $9 \mathrm{c}$ to $9 \mathrm{e}$, respectively, and therefore would be problematic for long term cycling.

A striking difference regards a large increase in polarization of the LTFS electrode from 200 to $500 \mathrm{mV}$ as $\mathrm{Li}_{0.5} \mathrm{In}$ is replaced by $\mathrm{Li}$ (figure 10a). It simply originates from the lower applied pressure $\left(45 \mathrm{~kg} \mathrm{~cm}^{-2}\right)$ used for running the Li-based cell as compared to the $\mathrm{In}_{0.5} \mathrm{Li}$ one $\left(1 \mathrm{t} \mathrm{cm}^{-2}\right)$. This equally explain the lower values of both the reversible capacity and coulombic efficiency ( $180 \mathrm{~mA} \mathrm{~h} \mathrm{~g}^{-1}$ and $93 \%$ at $1^{\text {st }}$ cycle) measured for the $\mathrm{LiCE}$ as compared to the $\mathrm{Li}_{0.5} \mathrm{In}$ : $\beta$-LPS one. Lastly, the polarization for the $\mathrm{Li}$ anode 
is at least two times greater than for the $\mathrm{In}_{0.5} \mathrm{Li}$ one, despite the low current density of $27 \mu \mathrm{A} \mathrm{cm}^{-2}$.

Unlike with $\mathrm{Li}_{0.5} \mathrm{In}$ : $\beta$-LPS, with $\mathrm{Li}$ anode the solid electrolyte loop is not clearly distinguished in figures 10b) to 10e), which implies that the impedance of the Li/electrolyte interface has contributions until the highest frequencies explored. Moreover, after cell's assembly, figure $10 \mathrm{~b}$ shows the onset of a characteristic intermediate frequency loop (apex at $12 \mathrm{kHz}$ ) while at lowest frequencies the imaginary part of the impedance remains close to 0 . Upon plating this loop almost disappears within the first 2 hours whereas a low frequency loop gradually grows. Upon stripping (figure 10c), the low frequency loop quickly disappears and gradual growth of the intermediate frequency loop is observed, in such way that the spectra are qualitatively similar to the initial one recorded before cycling. The same scheme is reproduced for the 2nd cycle, with fast disappearance of the intermediate frequency loop and growth of the low-frequency loop upon plating (figure 10d). The last three spectra with apex at $3.7 \mathrm{mHz}$ superimpose almost perfectly, which indicates that a steady state is reached. Nevertheless, at subsequent stripping (figure 10e) the intermediate frequency loop grows again to a significantly higher extent than at first cycle.

Overall, the three electrodes setup allows to follow the impedance of the Li anode during cycling while offering the feasibility to determine the regions that are affected by the plating or stripping process. Such distinction could not be made unambiguously with a symmetric $\mathrm{Li} / \mathrm{Li}$ cell as both plating and stripping occur simultaneously. Nevertheless, data recorded in symmetric cells can now benefit from our three electrode measurements imput that have identified specific frequency domains associated to plating and stripping processes. Needless to say that more work is needed to determine the underlying physico-chemical processes of the observed phenomena. At this stage 
one could hypothetize that the growth upon stripping of the $\mathrm{kHz}$ frequency loop range is associated to an increasing amount of porosity at the Li/electrolyte interface. In contrast, we believe the growth of the low frequency loop to be associated to formation of a resistive layer upon plating, however its fast disappearance as the current is reversed would need to be rationalised.

\section{Conclusion}

A three electrodes cell for all-solid-state systems was realised and tested with various combinations of cathode active materials, electrolytes and anodes. The cell is easily assembled, air-tight, displays stable RE voltage for hundreds of hours and allows to collect impedance spectra free of artefact loops. To the best of our knowledge, none of the three electrode all-solid state cells reported this far combine all these features.

The preliminary results reported here show drastic difference between oxide and sulphide based active materials, with oxides displaying complex impedance spectra in combination with either $\beta$-LPS or argyrodite electrolyte. Moreover, we provide greater insinghts on the science underlying the Li stripping-plating processes. This analytical advance is timely bearing in mind that great development that solid state batteries are presently enjoying.

\section{Acknowledgements}

We thank Bernhard Leube for the synthesis of argyrodite and LTFS, and Benjamin Hennequart for helpful discussions. JMT aknowledges the funding from the European Research Council (ERC grant project 670116-ARPEMA). 


\section{References}

1 J. Janek and W. G. Zeier, Nature Energy, 2016, 1, 1-4.

2 Y. Kato, S. Hori, T. Saito, K. Suzuki, M. Hirayama, A. Mitsui, M. Yonemura, H. Iba and R. Kanno, Nature Energy, 2016, 1, 16030.

3 S. Randau, D. A. Weber, O. Kötz, R. Koerver, P. Braun, A. Weber, E. Ivers-Tiffée, T. Adermann, J. Kulisch, W. G. Zeier, F. H. Richter and J. Janek, Nature Energy, 2020, 5, 259-270.

4 D. W. Dees, A. N. Jansen and D. P. Abraham, J. Power Sources, 2007, 174, 10011006.

5 S. Klink, E. Madej, E. Ventosa, A. Lindner, W. Schuhmann and F. L. Mantia, Electrochem. Commun., 2012, 22, 120-123.

6 M. Ender, J. Illig and E. Ivers-Tiffée, J. Electrochem. Soc., 2017, 164, A71-A79.

7 Y. J. Nam, K. H. Park, D. Y. Oh, W. H. An and Y. S. Jung, J. Mater. Chem. A, 2018, 6, 14867-14875.

8 J. Kasemchainan, S. Zekoll, D. Spencer Jolly, Z. Ning, G. O. Hartley, J. Marrow and P. G. Bruce, Nature Materials, 2019, 18, 1105-1111.

9 R. Schlenker, D. Stępień, P. Koch, T. Hupfer, S. Indris, B. Roling, V. Miß, A. Fuchs, M. Wilhelmi and H. Ehrenberg, ACS Applied Materials \& Interfaces, 2020, 12, 20012-20025.

10 S. Solchenbach, D. Pritzl, E. J. Y. Kong, J. Landesfeind and H. A. Gasteiger, J. Electrochem. Soc., 2016, 163, A2265-A2272.

11 A. Ikezawa, G. Fukunishi, T. Okajima, F. Kitamura, K. Suzuki, M. Hirayama, R. Kanno and H. Arai, Electrochemistry Communications, 2020, 116, 106743.

12 S. Saha, G. Assat, M. T. Sougrati, D. Foix, H. Li, J. Vergnet, S. Turi, Y. Ha, W. Yang, J. Cabana, G. Rousse, A. M. Abakumov and J.-M. Tarascon, Nature Energy, 2019, 4, 977-987.

13 Z. Liu, W. Fu, E. A. Payzant, X. Yu, Z. Wu, N. J. Dudney, J. Kiggans, K. Hong, A. J. Rondinone and C. Liang, Journal of the American Chemical Society, 2013, 135, 975-978.

14 C. Yu, S. Ganapathy, J. Hageman, L. van Eijck, E. R. H. van Eck, L. Zhang, T. Schwietert, S. Basak, E. M. Kelder and M. Wagemaker, ACS Appl. Mater. Interfaces, 2018, 10, 33296-33306.

15 J. Costard, M. Ender, M. Weiss and E. Ivers-Tiffée, J. Electrochem. Soc., 2017, 164, A80-A87.

16 F. Marchini, B. Porcheron, G. Rousse, L. Albero Blanquer, L. Droguet, D. Foix, T. Koç, M. Deschamps and J. M. Tarascon, Advanced Energy Materials, 2021, 2101111.

17 A. L. Santhosha, L. Medenbach, J. R. Buchheim and P. Adelhelm, Batteries \& Supercaps, 2019, 2, 524-529.

18 F. Marchini, S. Saha, D. Alves Dalla Corte and J. M. Tarascon, ACS Applied Materials \& Interfaces, 2020, 12, 15145-15154.

19 W. Zhang, T. Leichtweiß, S. P. Culver, R. Koerver, D. Das, D. A. Weber, W. G. Zeier and J. Janek, ACS Appl. Mater. Interfaces, 2017, 9, 35888-35896.

20 P. Zoltowski, Journal of Electroanalytical Chemistry, 1998, 443, 149-154.

21 I. D. Raistrick, D. R. Franceschetti and J. R. Macdonald, in Impedance Spectroscopy, John Wiley \& Sons, Ltd, 2005, pp. 27-128. 
22 W. Zhang, D. A. Weber, H. Weigand, T. Arlt, I. Manke, D. Schröder, R. Koerver, T. Leichtweiss, P. Hartmann, W. G. Zeier and J. Janek, ACS Applied Materials \& Interfaces, 2017, 9, 17835-17845.

23 N. Kaiser, S. Spannenberger, M. Schmitt, M. Cronau, Y. Kato and B. Roling, Journal of Power Sources, 2018, 396, 175-181.

24 Z. Siroma, T. Sato, T. Takeuchi, R. Nagai, A. Ota and T. Ioroi, Journal of Power Sources, 2016, 316, 215-223.

25 Y. Xiao, Y. Wang, S.-H. Bo, J. C. Kim, L. J. Miara and G. Ceder, Nature Reviews Materials, , DOI:10.1038/s41578-019-0157-5.

26 G. Assat and J.-M. Tarascon, Nat Energy, 2018, 3, 373-386.

27 A. Tsuchimoto, X.-M. Shi, K. Kawai, B. Mortemard de Boisse, J. Kikkawa, D. Asakura, M. Okubo and A. Yamada, Nat Commun, 2021, 12, 631.

28 M. S. Whittingham, Prog. Solid State Chem., 1978, 12, 41-99.

29 B. R. Shin, Y. J. Nam, D. Y. Oh, D. H. Kim, J. W. Kim and Y. S. Jung, Electrochimica Acta, 2014, 146, 395-402. 
a)

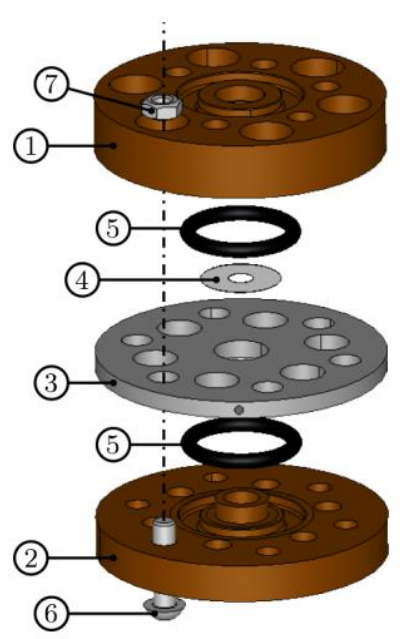
(1) : half body 1
(2) : half body 2
(3) : RE collector
(4) : metal foil
(5) : o-ring
(6) : screw

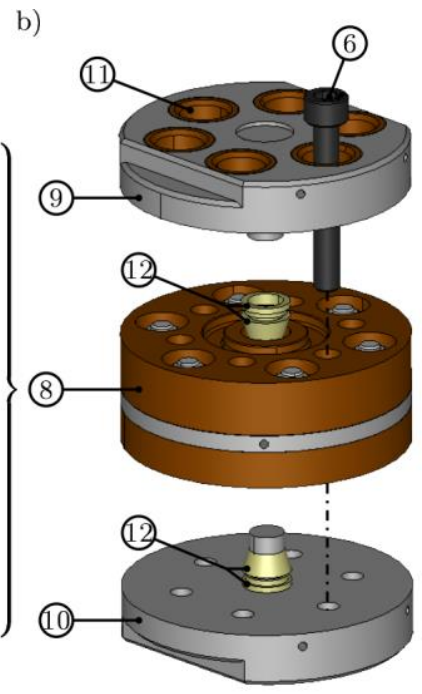

(7) : nut

(8) : complete cell body

(9) : top platen

(10) : bottom platen

(11) : insulator

(12) : ferrules

Figure 1: the 3 electrodes cell hardware: a) body; b) complete cell. 
a)

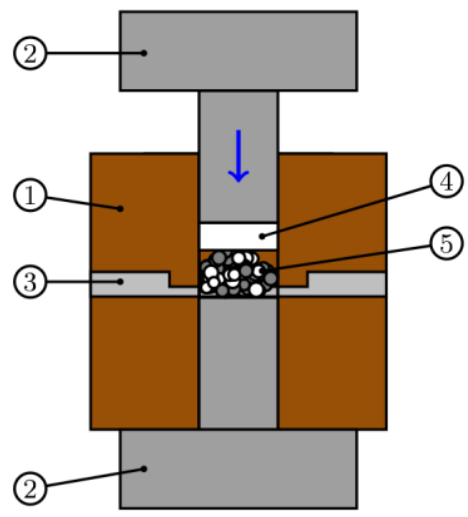

c)

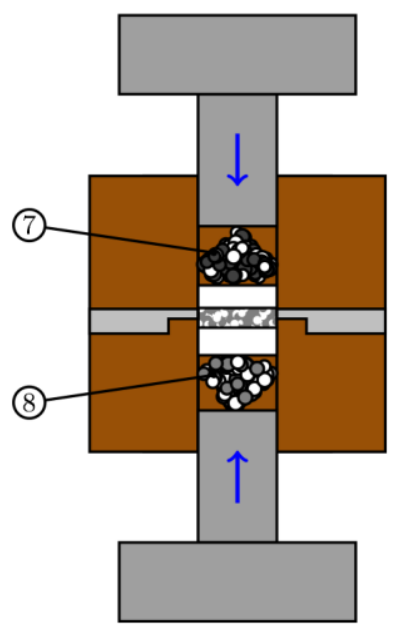

b)

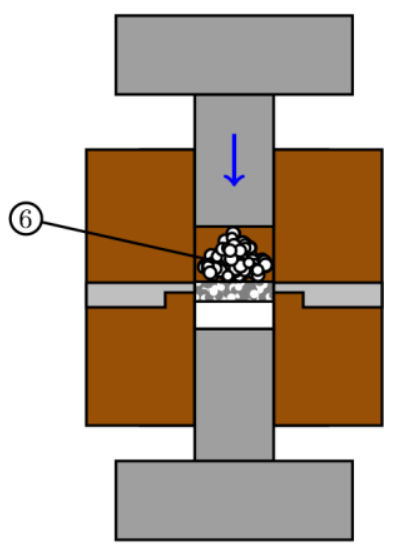

d)

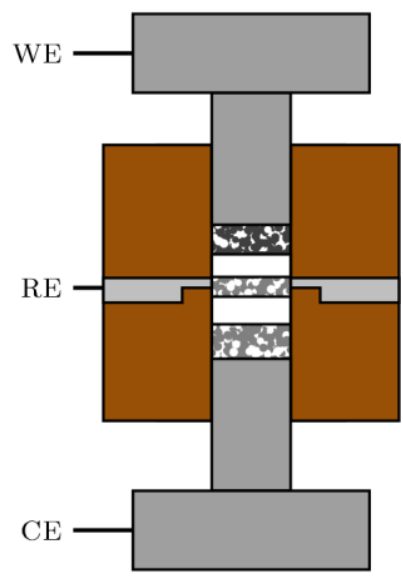
(1) cell body
(2) piston
(3) RE current collector
(4) electrolyte pellet

(5) RE powder

(6) electrolyte powder

(7) WE powder

(8) CE powder

Figure 2: assembly procedure of the 3 electrodes all solid state cell: a) assembly of the reference layer with a solid electrolyte pellet; $b$ ) addition of a 2nd electrolyte layer; c) addition of working and counter electrodes; d) cell ready to cycle. Blue arrows indicate the displacement of mobile pistons w./ respect to the cell body at each step. 

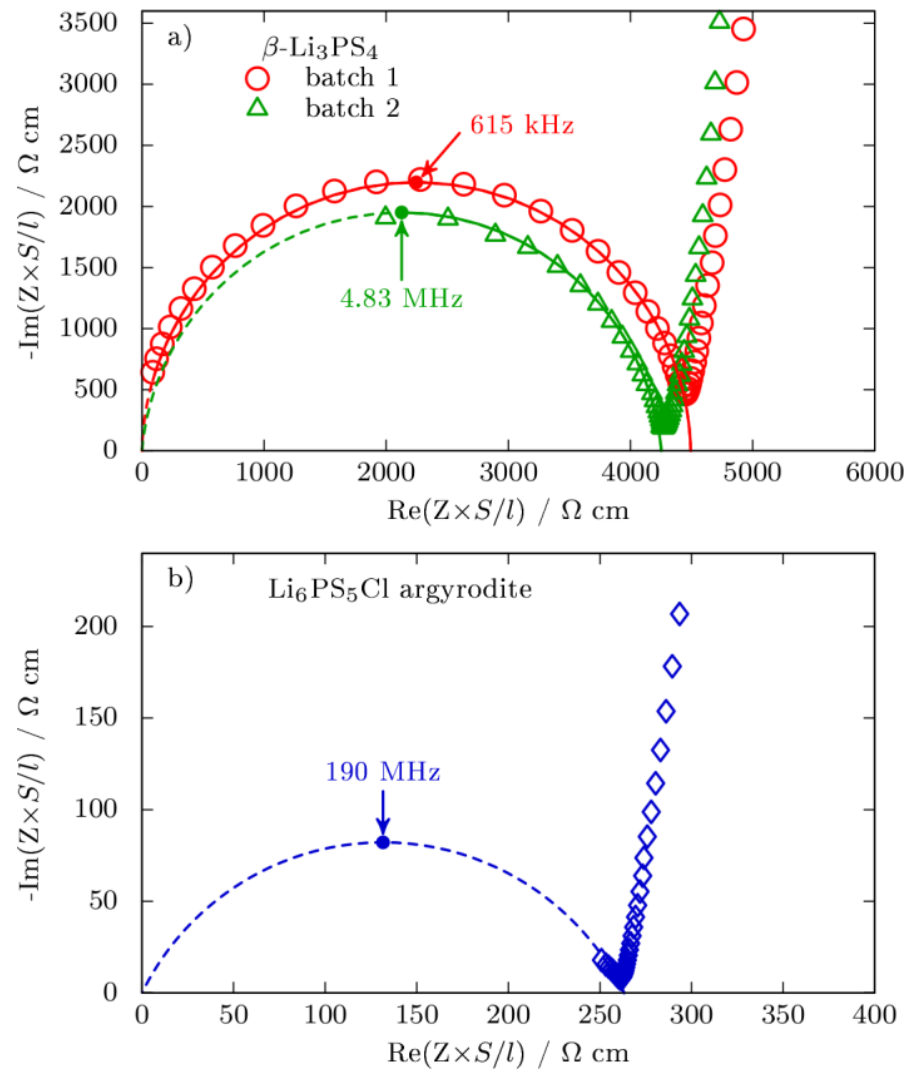

Figure 3: complex resistivity of solid electrolyte pellets: a) b-Lii $\mathrm{PS}_{4}$ and b) $\mathrm{Li}_{6} \mathrm{PS}_{5} \mathrm{Cl}$. The high frequency response is fitted with the impedance of a parallel R-CPE circuit (solid lines) and extrapolated until $\mathrm{Z}=0$ at infinite frequency (dashed line). 

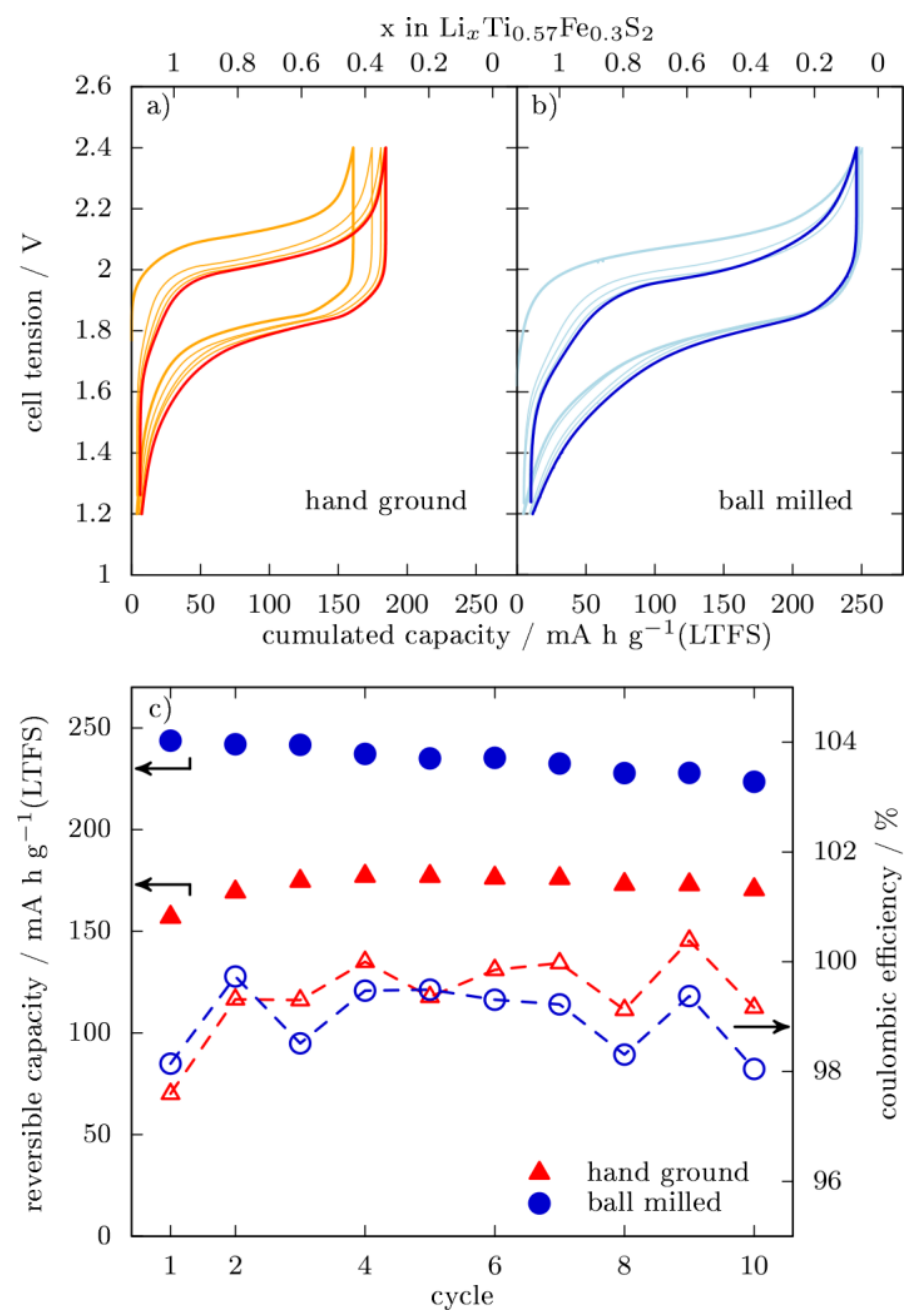

Figure 4: voltage profile of LTFS $v s$. $\mathrm{Li} 0.5 \mathrm{In}$ anode for hand ground composite (a) and ball-milled composite (b) at $\mathrm{C} / 25$; reversible capacity and coulombic efficiency of both cells (c). 

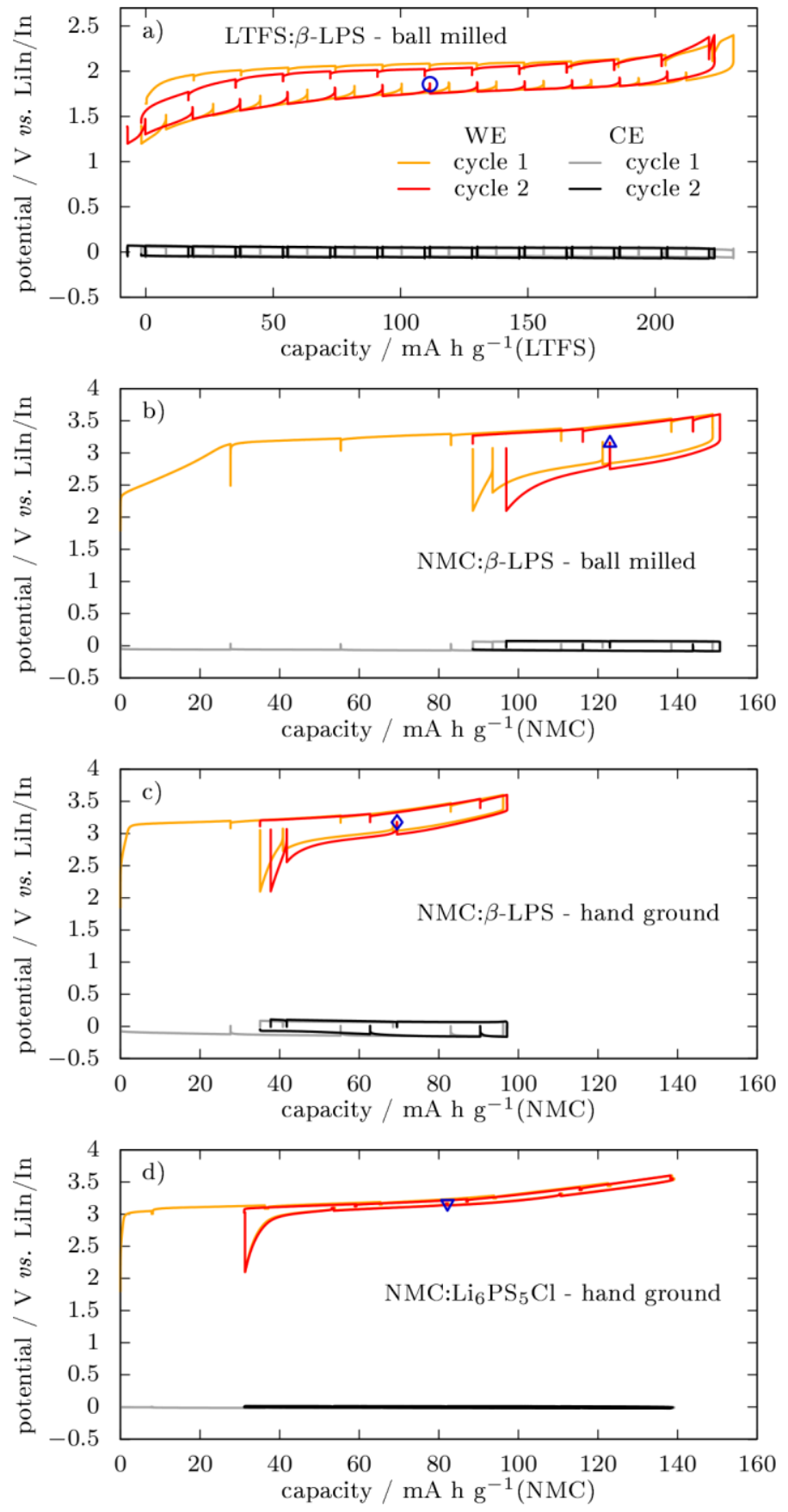

Figure 5: galvanostatic voltage profiles of $\mathrm{WE}$ and $\mathrm{Li}$ 0.5 In $\mathrm{CE}$ for the first 2 cycles in 3 electrodes configuration. WE active material is LTFS (a) or $\mathrm{NMC}_{622}$ (c-d); WE composites are prepared by high-energy ball milling (a-b) or hand grinding (c-d); electrolyte is $\beta-\mathrm{Li}_{3} \mathrm{PS}_{4}(\mathrm{a}-\mathrm{c})$ or $\mathrm{Li}_{6} \mathrm{PS}_{5} \mathrm{Cl}$ argyrodite $(\mathrm{d})$. 

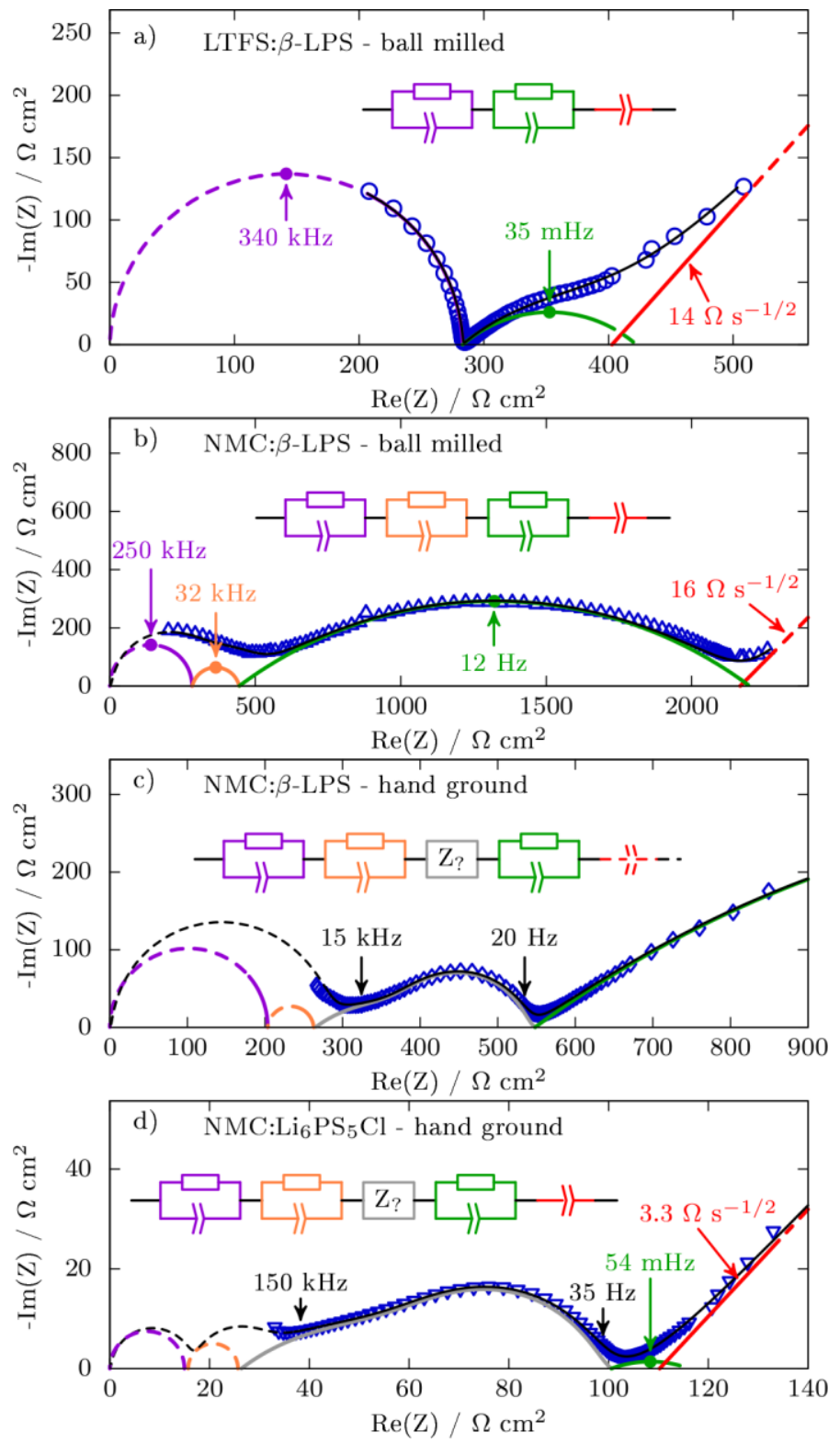

Figure 6: impedance of the various WE composites recorded at the middle of $2^{\text {nd }}$ reduction: a) ball-milled LTFS: $\beta$-LPS; b) ball-milled NMC622: $\beta$-LPS; c) hand ground NMC622: $\beta$-LPS; d) hand ground NMC622: $\operatorname{Li}_{6} \mathrm{PS}_{5} \mathrm{Cl}$. A black line indicates the fit of the spectra with a series of parallel R-CPE elements depicted in each subfigure. The impedances of their constitutive elements are plotted with their respective colour. In c) and d) $Z_{\text {? }}$ is a series of two R-CPE elements. 


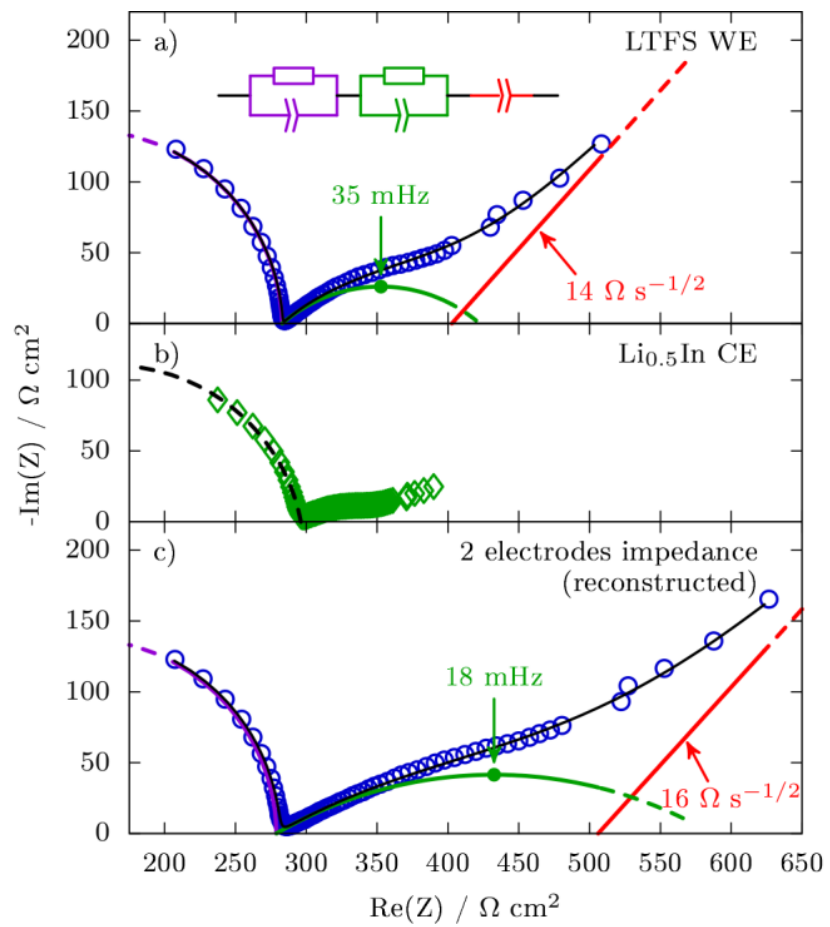

Figure 7: a) impedance of partially lithiated LTFS recorded in the middle of 2nd discharge (open symbols); b) impedance of the $\mathrm{Li}_{0.5}$ In counter electrode simultaneously recorded; c) impedance of a 2 electrodes LTFS:Li ${ }_{0.5}$ In cell as reconstructed from the spectra in a) and b). The spectra in a) and c) are fitted using the equivalent circuit displayed in a) (solid lines). 

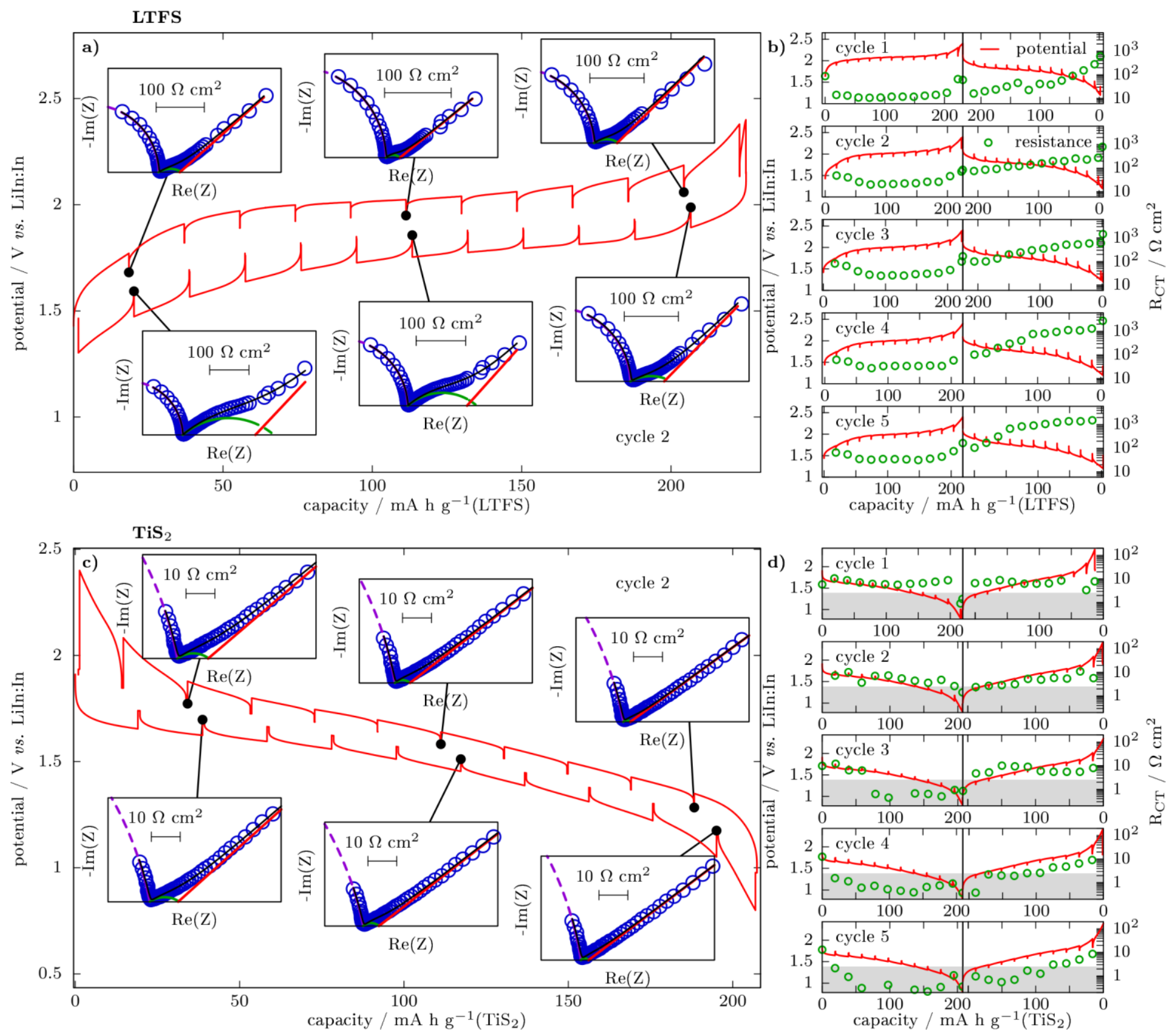

Figure 8: 2nd cycle and variations of $\mathrm{R}_{\mathrm{CT}}$ during cycling for LTFS ( a) and b), respectively) and $\mathrm{TiS}_{2}$ (c and d). Insets in a) and c) report the impedance spectra of LTFS and $\mathrm{TiS}_{2}$ at various states of charge during oxidation and reduction. 

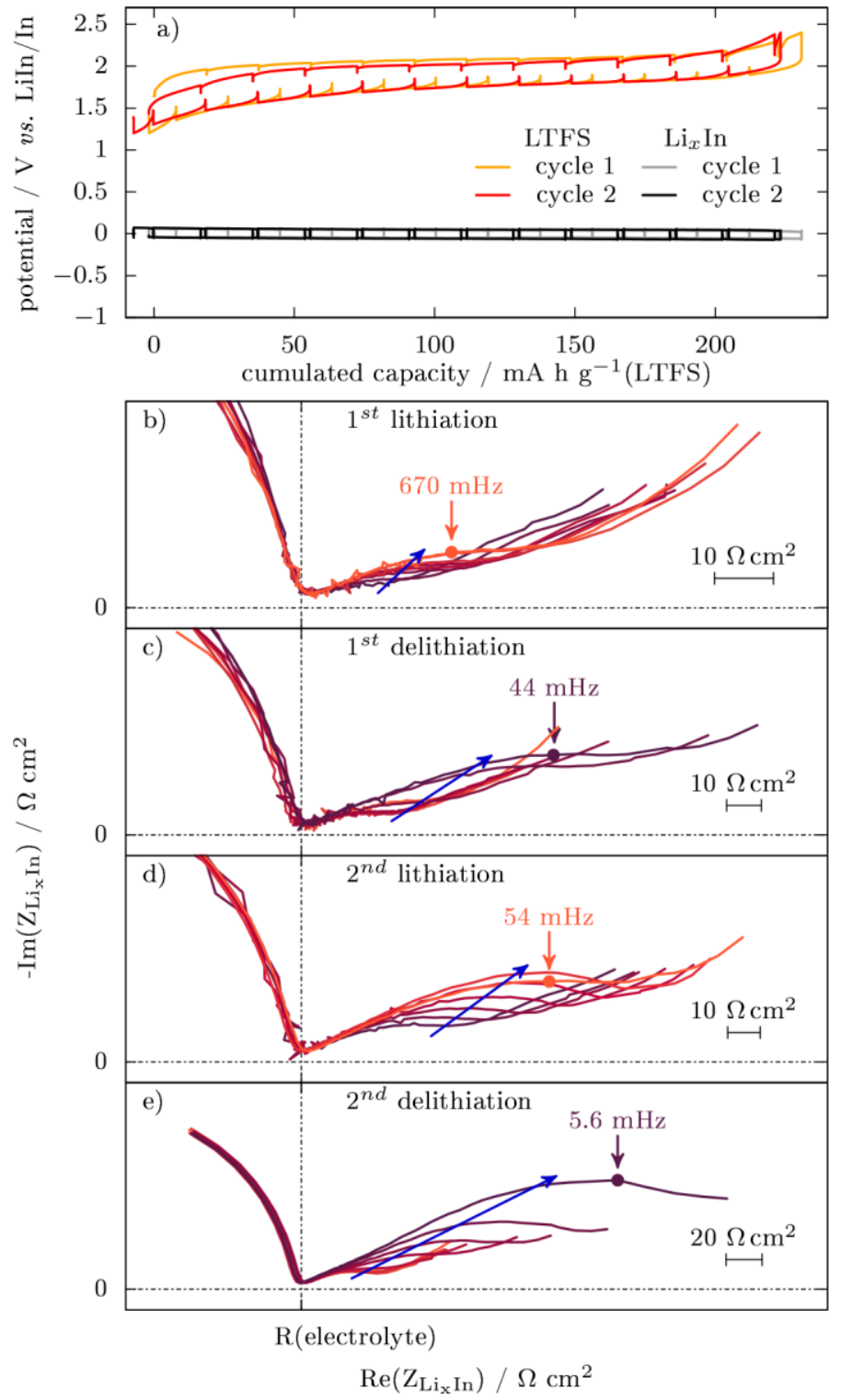

Figure 9: galvanostatic voltage profiles of LTFS WE and Li $0.5 \mathrm{In} \mathrm{CE}$ under $1 \mathrm{t} \mathrm{cm}^{-2}$ (a) for the first 2 cycles; impedance of the Li-In alloy CE (b-e) for each charge and discharge sequence. Blue arrows indicate the evolution of the impedance at each sequence. 

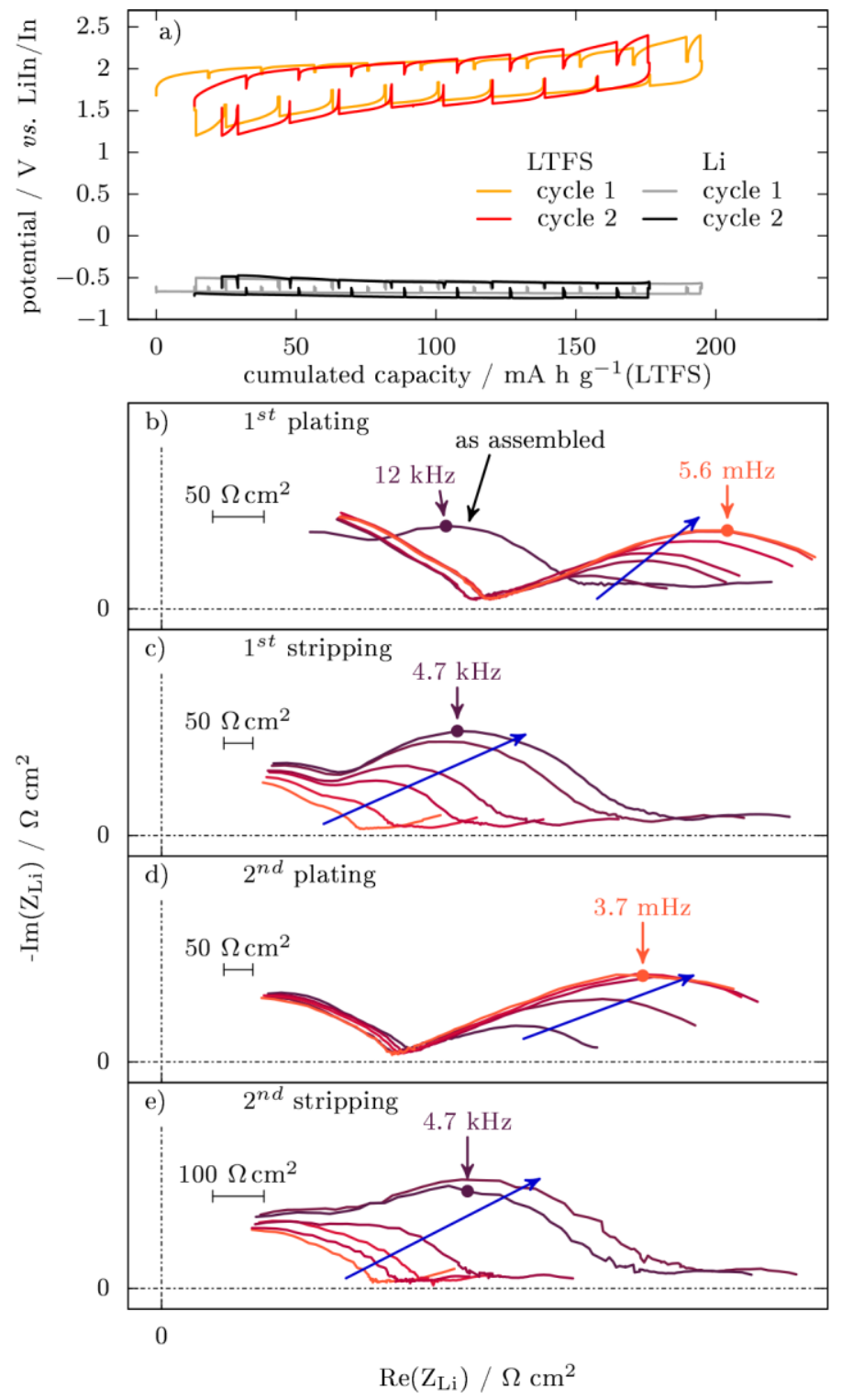

Figure 10: galvanostatic voltage profiles of LTFS WE and Li CE (a) under $45 \mathrm{~kg} \mathrm{~cm}^{-2}$ for the first 2 cycles; impedance of the Li metal CE (b-e) for each charge and discharge sequence. Blue arrows indicate the evolution of the impedance at each sequence. 